\title{
Aspect ratio effect on shear modulus and ultimate shear strength of
}

\section{graphene nanoribbons}

\author{
Tarek Ragab ${ }^{1}$, Julia McDonald ${ }^{2}$, Cemal Basaran ${ }^{2, *}$ \\ ${ }^{1}$ Arkansas State University, State University, AR, 72467 \\ ${ }^{2}$ Electronic Packaging Laboratory, University at Buffalo, New York, 14260
}

\begin{abstract}
This paper focuses on shear modulus and shear deformation behavior of Graphene Nano Ribbons (GNR) post wrinkling initiation taking into account size effect. Atomistic modeling of armchair and zigzag GNR were performed at $300^{\circ} \mathrm{K}$ for GNR with a constant $10 \mathrm{~nm}$ width and lengths ranging from $2.5 \mathrm{~nm}$ to $25 \mathrm{~nm}$. Results show that ridge formation is oriented at 45 degrees for GNR with an aspect ratio less than one and that the number of ridges is equal to the reciprocal of the aspect ratio, while for aspect ratios higher than one, the ridge formation direction coincides with the diagonal direction of the GNR and only one ridge is formed. Once the diagonal tension reached its ultimate elastic level, three major stress-relaxing phenomena are reported. Results show that the average slope of the stress-strain curve beyond the ultimate elastic stress decreases with increasing GNR length. Moreover, the slope of the shear stress-strain curve in that region is always greater in armchair GNR than in Zigzag GNR. GNR can sustain very high plastic shear strains beyond $400 \%$ before failure for wide GNR. It is observed that the shear modulus is inversely proportional to the aspect ratio of the GNR.
\end{abstract}

\section{Keywords}

Molecular dynamics simulations; Shear strength; Unravelling; Wrinkling; Buckling; GNR

Corresponding author ${ }^{*} \underline{c j b @ b u f f a l o . e d u}$ 


\section{Introduction}

Since the first successful exfoliation of the single atom-thick graphene in 2004 [1], it attracted intense research interest due to extraordinary electrical $[2,3]$, thermal $[4,5]$ and mechanical $[6$, 7] properties. Graphene Nanoribbons (GNR) which are finite nano-scale wide graphene are also a promising material for nano-electronics applications. However, their electrical and mechanical properties can vary widely depending on chirality and dimensions [8,9]. Moreover, experimental data shows great disparity due to the fact that it is very difficult to determine defect density and chirality of the tested samples.

Mechanically, GNR have been studied extensively for their tensile properties both experimentally[10,11] and analytically[12,13]. Only very few studies have been done on the shear behavior of GNR [14-16] and functionalized GNR [17, 18]. All of these studies use Molecular Dynamics (MD) simulations, which is a widely accepted method for modeling the mechanical and thermal properties of nanoscale materials. MD simulations were also used to study the size effect in different materials successfully [19-21]. Previous studies done to study GNR under shear deformation focused only on the behavior until wrinkling initiation (as shown in Figure 1). Min and Aluru [14] reported the shear stresses until wrinkling initiation while other papers focused only on other aspects regarding the ridge formation strain, ridge height and half wavelength of the ridge. Wang [16] studied the effect of the aspect ratio in the range from 1 to 10 and then to infinity by having periodic boundary conditions, but they didn't report the stressstrain relation and how it is affected by the aspect ratio as well as the plastic deformations that happen beyond the wrinkling stage. Therefore, this is the first study in the literature that focuses on shear modulus and shear deformation until failure and also take into account the aspect ratio. 
MD simulations were used to investigate armchair and zigzag GNR with different aspect ratios beyond wrinkling and the formation of permanent (plastic) damage and a deep understanding of the behavior under shear deformation is obtained. In the subsequent section, details of the MD simulations are given, and then full analysis of the shear behavior and results are given in the later section.
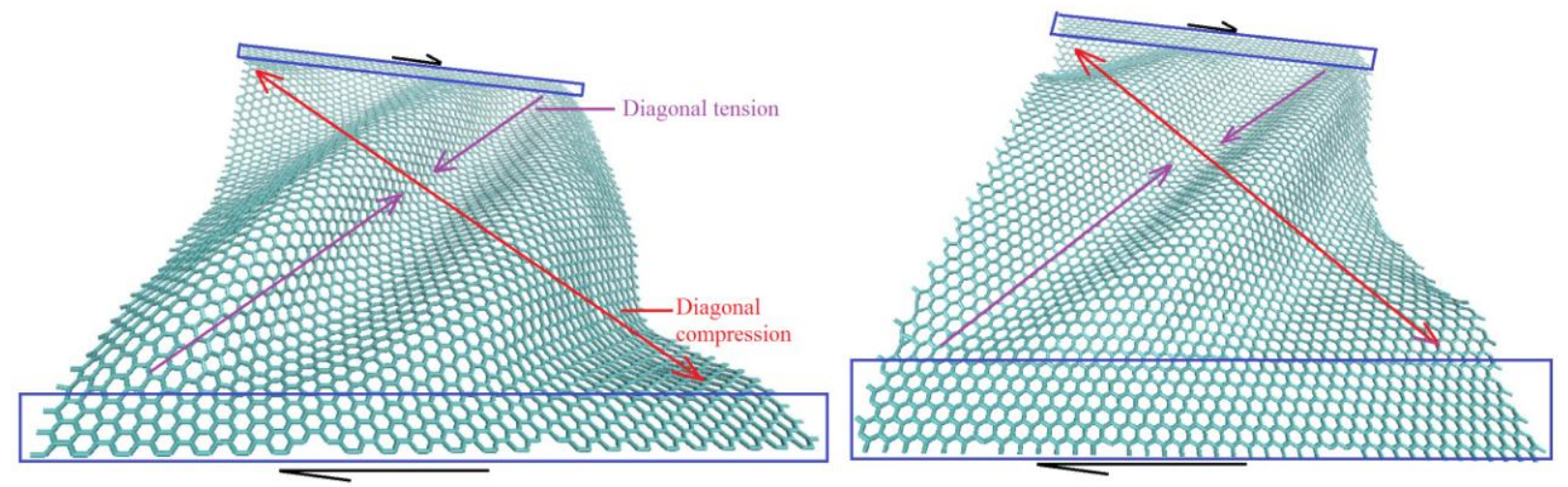

Figure 1 Illustration of the wrinkling effect in 10nm-square zigzag (left) and armchair (right) GNR under shear deformation

\section{Molecular Dynamics Simulations}

MD simulations were performed on armchair and zigzag GNR with fixed width of $10 \mathrm{~nm}$ under in-plane shear with no twisting[22] deformation until $10 \mathrm{~nm}$ of shear deformation was attained. The second generation Reactive Empirical Bond Order (REBO)[23] potential was used due to its ability to take into account the generation and breakage of bonds as well as rehybridization. Simulations were performed in the canonical (NVT) ensemble using Brendesen thermostat 
technique[24] for all the atoms to keep the simulation temperature constant at $300^{\circ} \mathrm{K}$. The integration of the equation of motion was done using the third order predictor-corrector algorithm with a time step of 0.25 fs which is less than $5 \%$ of the vibrational period for carbon atoms[25]. GNR were simulated with different aspect ratios ranging from 0.25 to 2.5 equivalent to lengths ranging from $2.5 \mathrm{~nm}$ to $25 \mathrm{~nm}$. In order to simulate the shear deformation, three unit cells were added on two opposite sides of the GNR as shown with the enclosed rectangles in Figure 1. Atoms in these regions were not allowed to move freely according to the equation of motion, but instead, upper atoms were moved to the right and lower atoms were moved to the left according to a prescribed displacement increment of 0.01 angstroms (which is a value proven to not cause any computational error[26]). By this prescribed displacement, only in-plane shear deformation is allowed without any twisting. The prescribed displacement was applied

once every 200 time steps. Thus the strain rate is varying from $8 \mathrm{E} 8 \mathrm{~s}^{-1}$ for the $25 \mathrm{~nm}$ long GNR to $8 \mathrm{E} 9 \mathrm{~s}^{-1}$ for the $2.5 \mathrm{~nm}$ long GNR. In order to calculate the shear stress during the application of the shear deformation virial stresses were used. In order to calculate the volume the thickness of the GNR was taken equal to $0.3335 \mathrm{~nm}$ [27]. Stresses were averaged over 200 consecutive time steps.

\section{Results and Discussion}

MD Simulations were performed for $10 \mathrm{~nm}$ wide armchair and zigzag GNR with $2.5 \mathrm{~nm}$, 5nm, $7.5 \mathrm{~nm}, 10 \mathrm{~nm}, 15 \mathrm{~nm}, 20 \mathrm{~nm}$ and $25 \mathrm{~nm}$ lengths not including the constrained boundary atoms. The stress strain curves for zigzag and armchair GNR are plotted in Figure 2 and Figure 3, respectively. It is observed that, all stress-strain curves pass through certain key points. First, the stress-strain curve evolves monotonically with the increasing shear displacement and during that period, wrinkling takes place and deformation is completely elastic in the sense that no bond 
formation, bond breakage or re-hybridization occur. It is important to note here that the shear deformation results in two diagonal forces; one is tensile and the other is compressive as illustrated in Figure 1. As opposed to what can be assumed[14, 15] that the wrinkling is the key factor affecting the behavior of GNR under shear deformation, it is actually the diagonal tension and its evolution, relaxation and interaction with the ridges in the perpendicular direction, while the compressive force in the diagonal compressive direction is almost zero and has no effect on the mechanics. Another important factor that affects the behavior is the flexure moment generated by the induced shear that becomes significant with increasing aspect ratio. After the diagonal tension reaches its ultimate elastic capacity, defects start to generate in the diagonal direction and stress-relaxing phenomena occurs. One of the stress-relaxing phenomena is the addition of atoms to the diagonal tension direction from neighboring locations, thus increasing the length in that direction and relieving a part of the stress similar to unraveling phenomenon in carbon nanotubes [28]. Another stress-relaxing phenomenon is the internal nucleation of defect at the ridge edge. The third stress-relaxing phenomenon involves the breakage of bonds at the end of the major diagonal tension direction and thus sliding that major diagonal tension direction towards the inside of the GNR and thus decreasing the stress. This specific phenomenon is usually accompanied by a decrease in the ability of the GNR to build a higher stress due to the decrease in the effective length of the GNR edge due to the breakage of bonds. These stress relaxing phenomena are illustrated in Figure 4 through Figure 9, which show the evolution of the shear deformation in $2.5 \mathrm{~nm}, 10 \mathrm{~nm}$ and $25 \mathrm{~nm}$ long zigzag and armchair GNR respectively. It is clear from the figures that for aspect ratios less than one (wide GNR) the angle of the ridge formation is always equal to 45 degrees (in the direction of the diagonal tension) and the number of ridges formed is equal to the inverse of the aspect ratio as shown in Figure 4 and Figure 7 . The 
reader is encouraged to refer to figure 8 of reference [16] for wider GNRs. When the aspect ratio is more than one (in long GNR) only one ridge is formed in the direction of the diagonal which is more than 45 degrees and the angle increases with the increasing aspect ratio.

While the size of the GNR has no effect on the shear modulus in a square GNR, it is clear from Figure 10 that increasing the aspect ratio from 0.25 to 2.5 will decrease the shear modulus from $138 \mathrm{GPa}$ to $59 \mathrm{GPa}$ in zigzag GNR and from $96 \mathrm{GPa}$ to $36 \mathrm{GPa}$ in armchair GNR. It is worth mentioning that increasing the aspect ratio has a more profound effect on the modulus of elasticity at higher values than at lower values especially for aspect ratios greater than one. Because when a GNR is long, the bending moment induced by the shear force becomes larger. As a result GNR is subjected to a combined bending moment and a shear force. Hence, bending contributes to softening of the GNR, which is more significant at higher aspect ratios[ longer the GNR bigger the aspect ratio]. Bending also contributes to the direction of the ridge formation as explained in the previous paragraph. It is also worth mentioning that the effect of the aspect ratio on the uniaxial properties of armchair and zigzag GNR has been reported before[7] and results show no effect at all on the modulus of elasticity. Thus it is fairly stated that the dependence of the shear modulus on the aspect ratio reported here cannot be related to a change in the uniaxial properties along the direction of the maximum tensile force. 


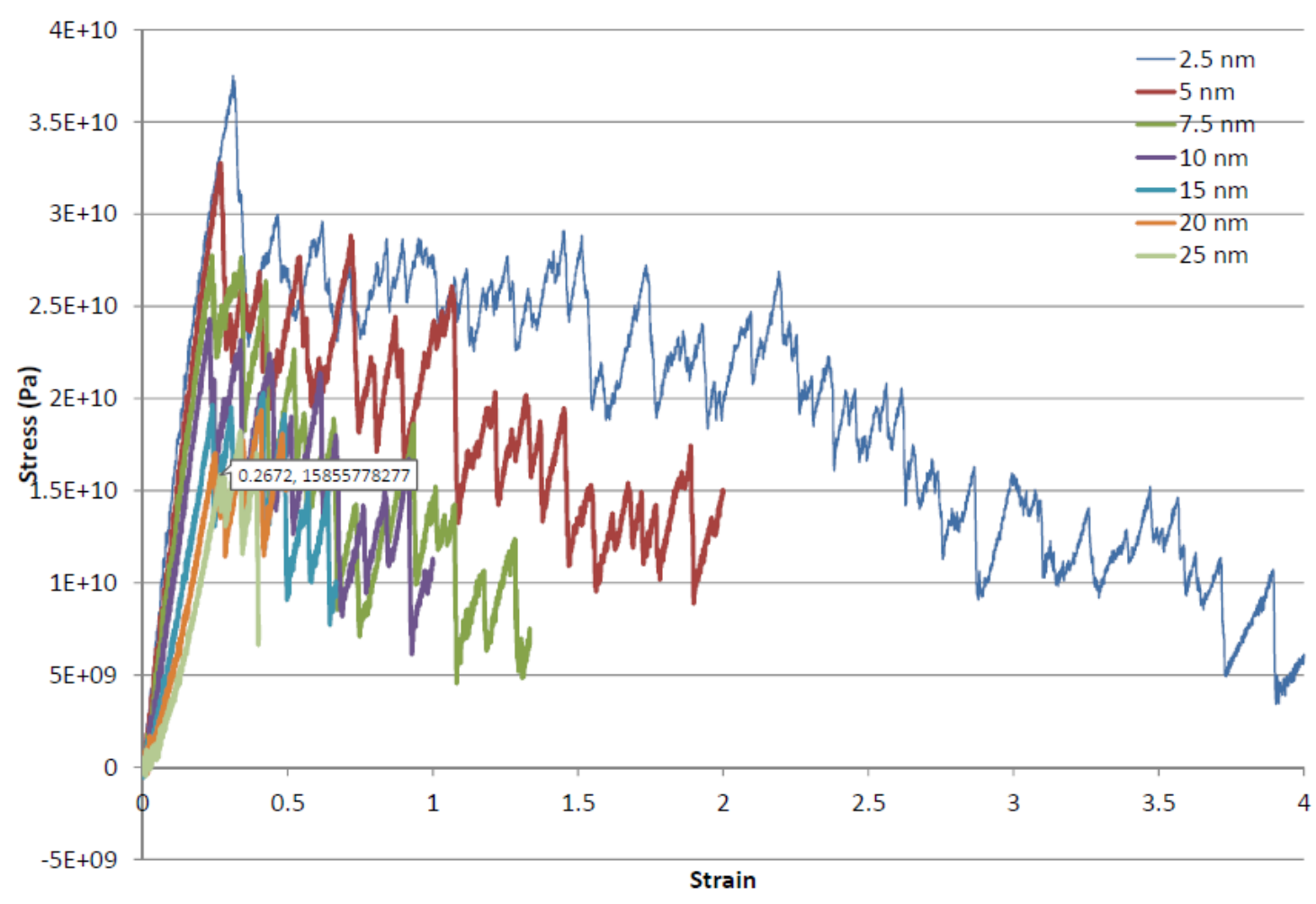

Figure 2 Stress-strain curves for zigzag GNR with different lengths

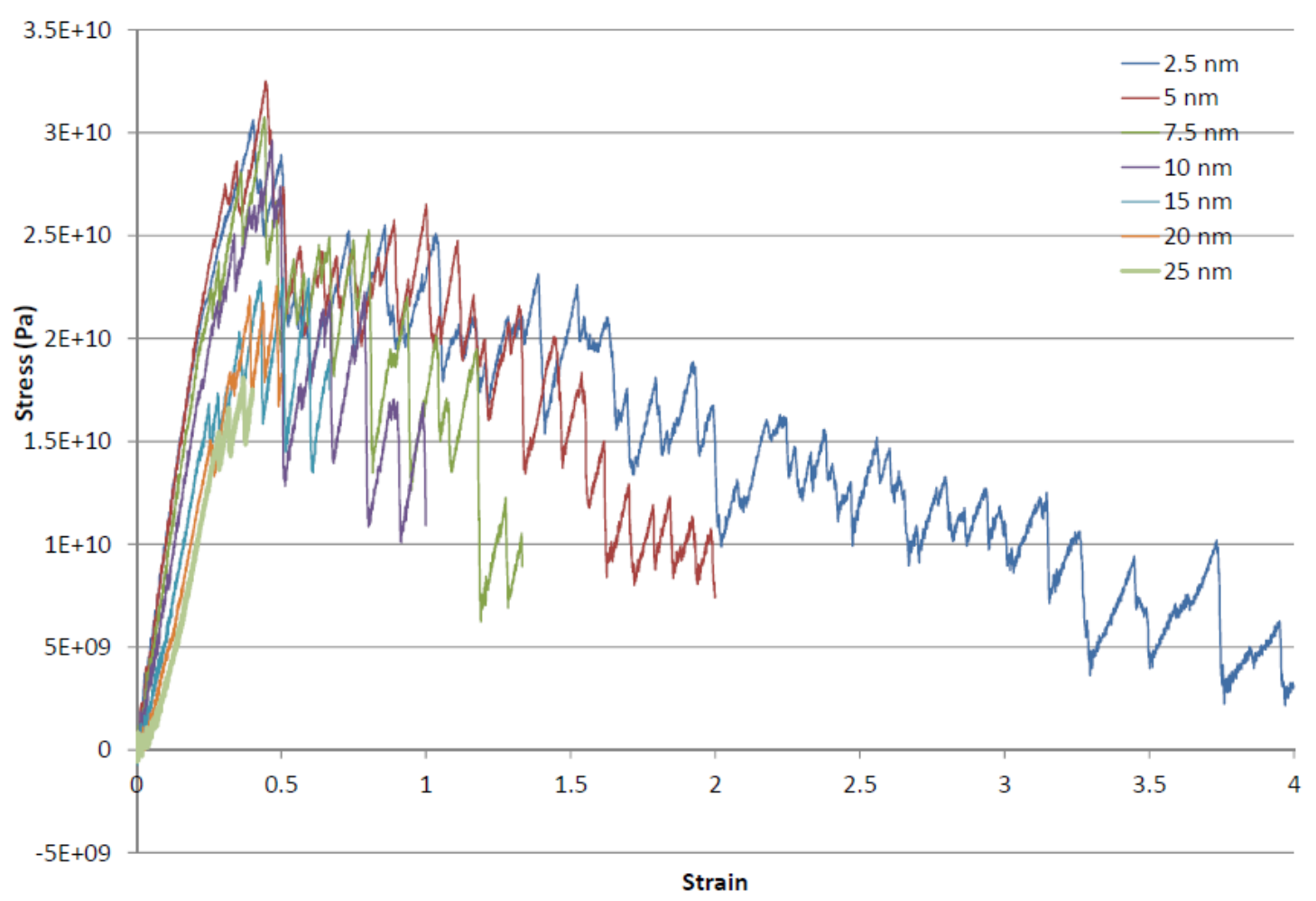

Figure 3 Stress-strain curves for armchair GNR with different lengths 
Longer the GNR, there is more probability for stress relaxation to occur by breaking of the bonds in the tension zone, in the post-ultimate stress point. This is true for both for armchair and zigzag GNR. This stress relaxation by breaking bonds leads to a decrease in the effective width, which in turn results in the monotonic decrease of stress as shown in Figure 2 and Figure 3. Moreover, as discussed, this effect decreases with increasing aspect ratio for values higher than one.
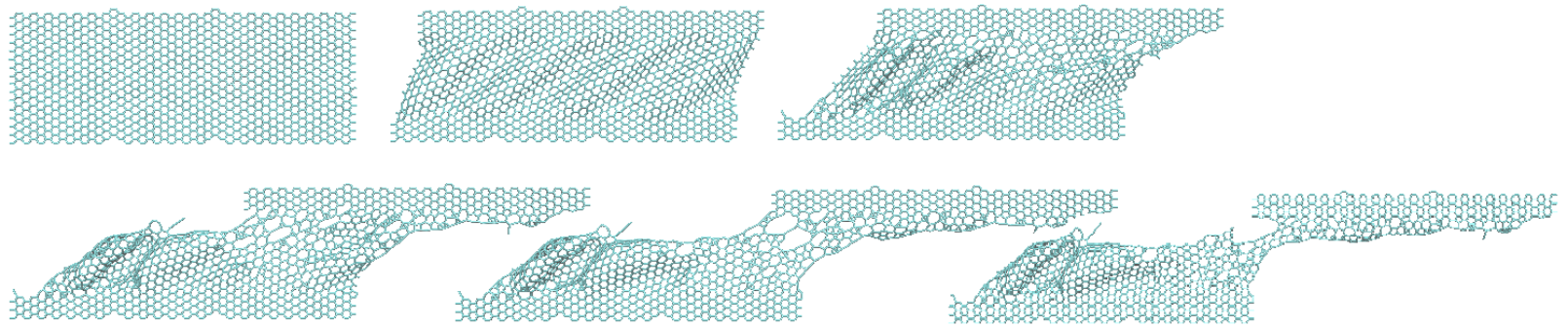

Figure 4 Evolution of the shear deformation in $2.5 \mathrm{~nm}$ long zigzag GNR until 400\% strain
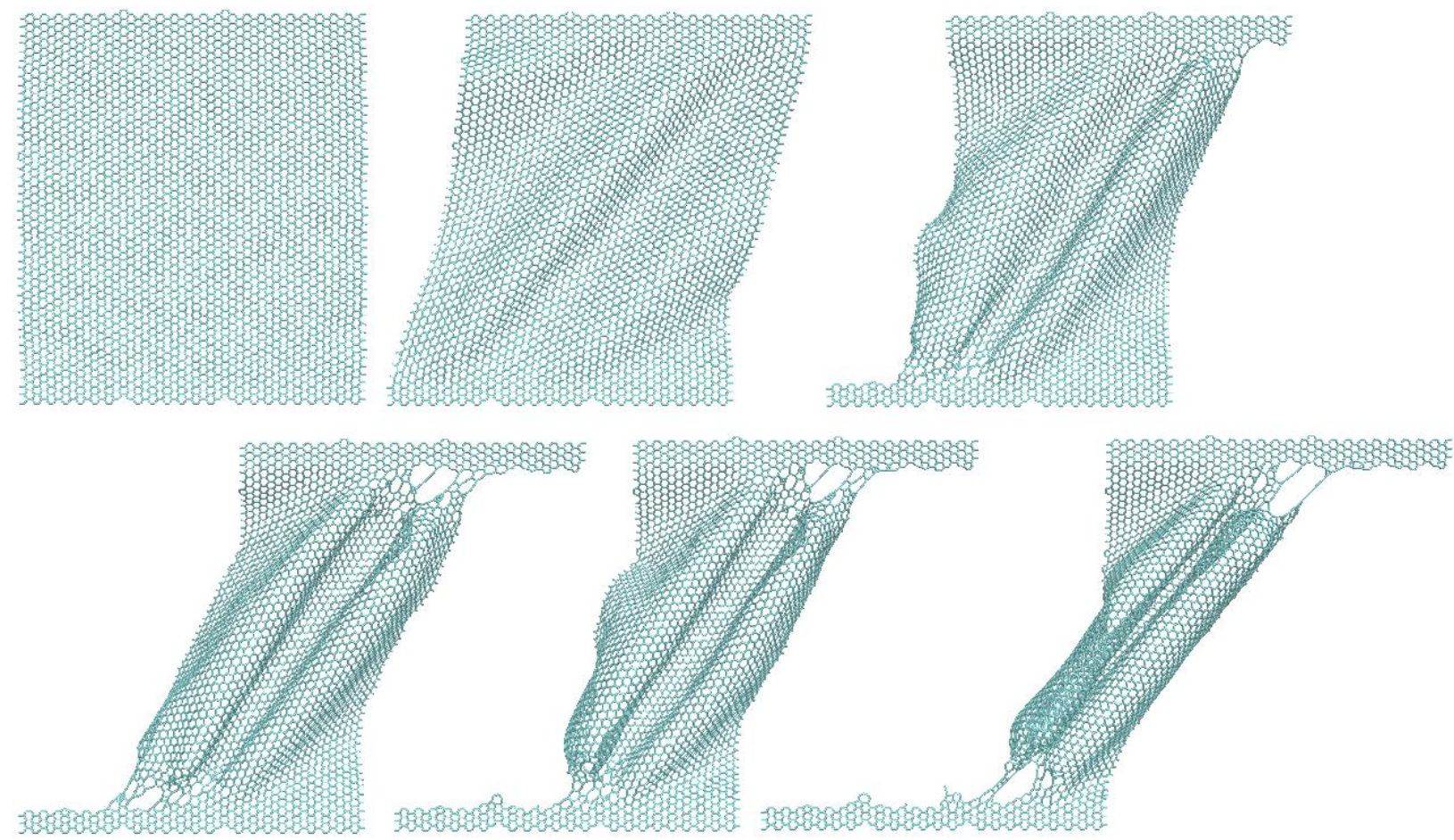

Figure 5 Evolution of the shear deformation in $10 \mathrm{~nm}$ long zigzag GNR until 100\% strain 


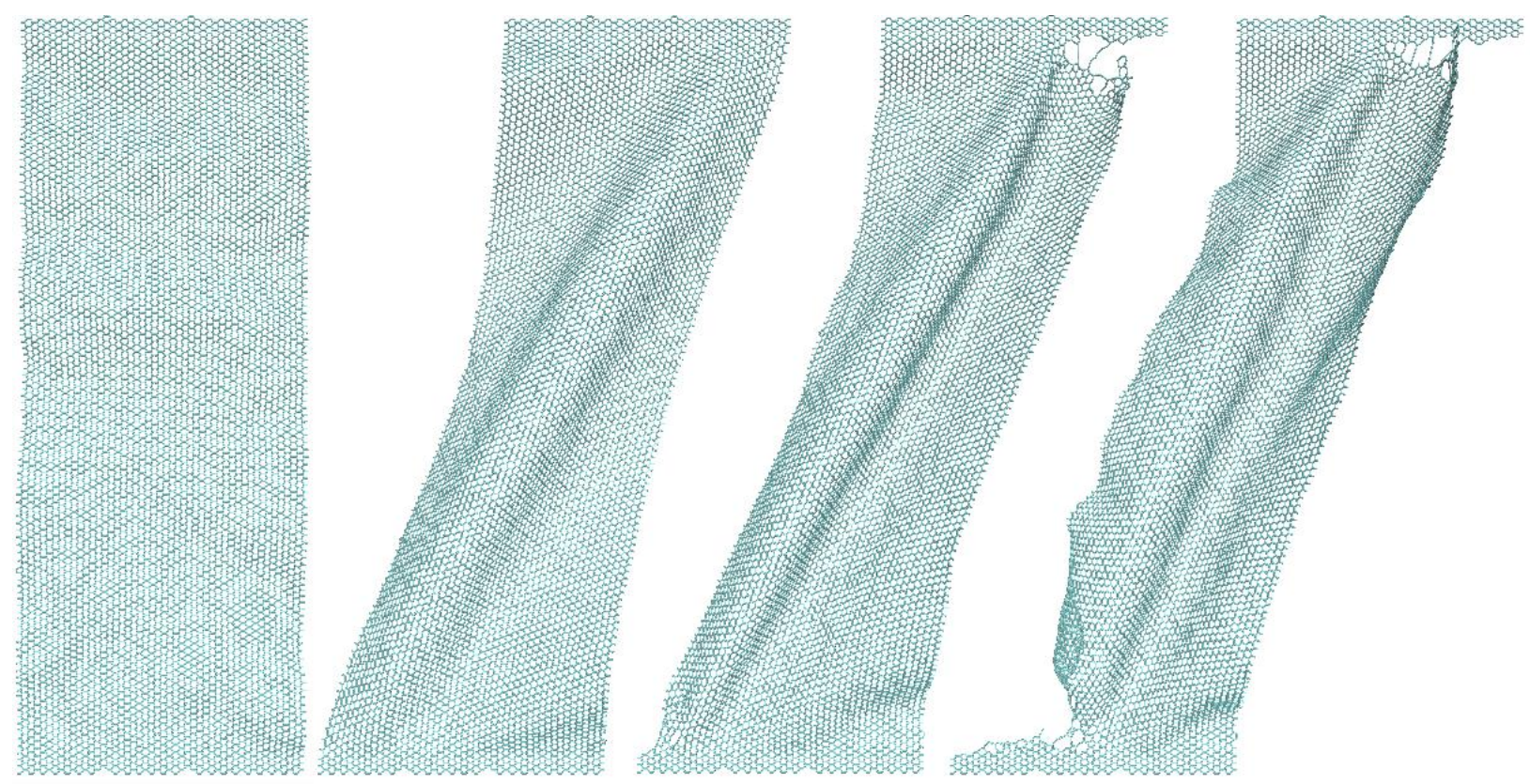

Figure 6 Evolution of the shear deformation in $25 \mathrm{~nm}$ long zigzag GNR until $40 \%$ strain
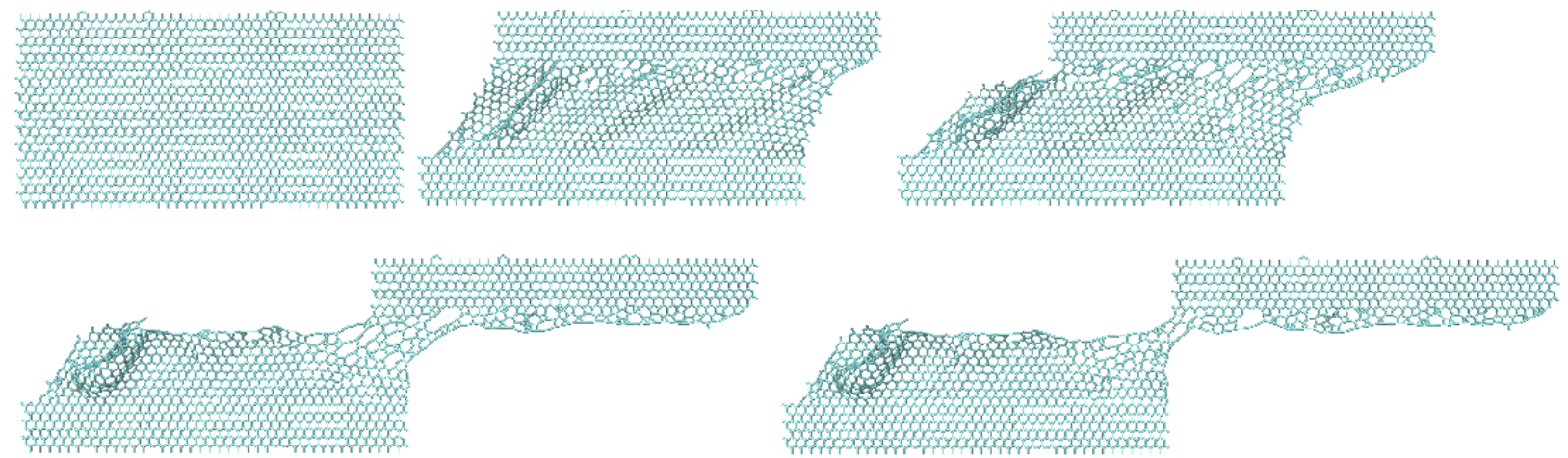

Figure 7 Evolution of the shear deformation in $2.5 \mathrm{~nm}$ long armchair GNR until $400 \%$ strain 

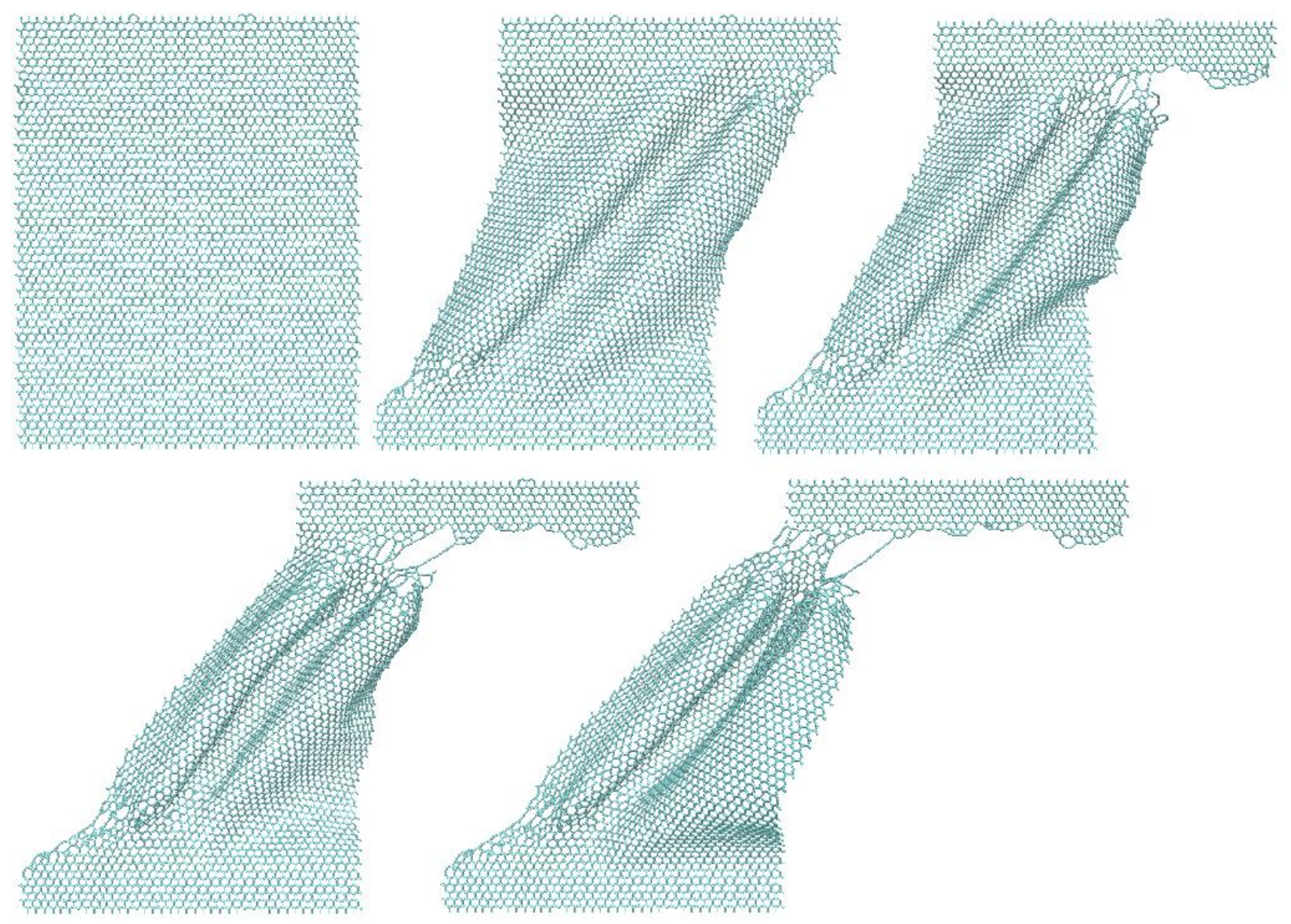

Figure 8 Evolution of the shear deformation in $10 \mathrm{~nm}$ long armchair GNR until 100\% strain 

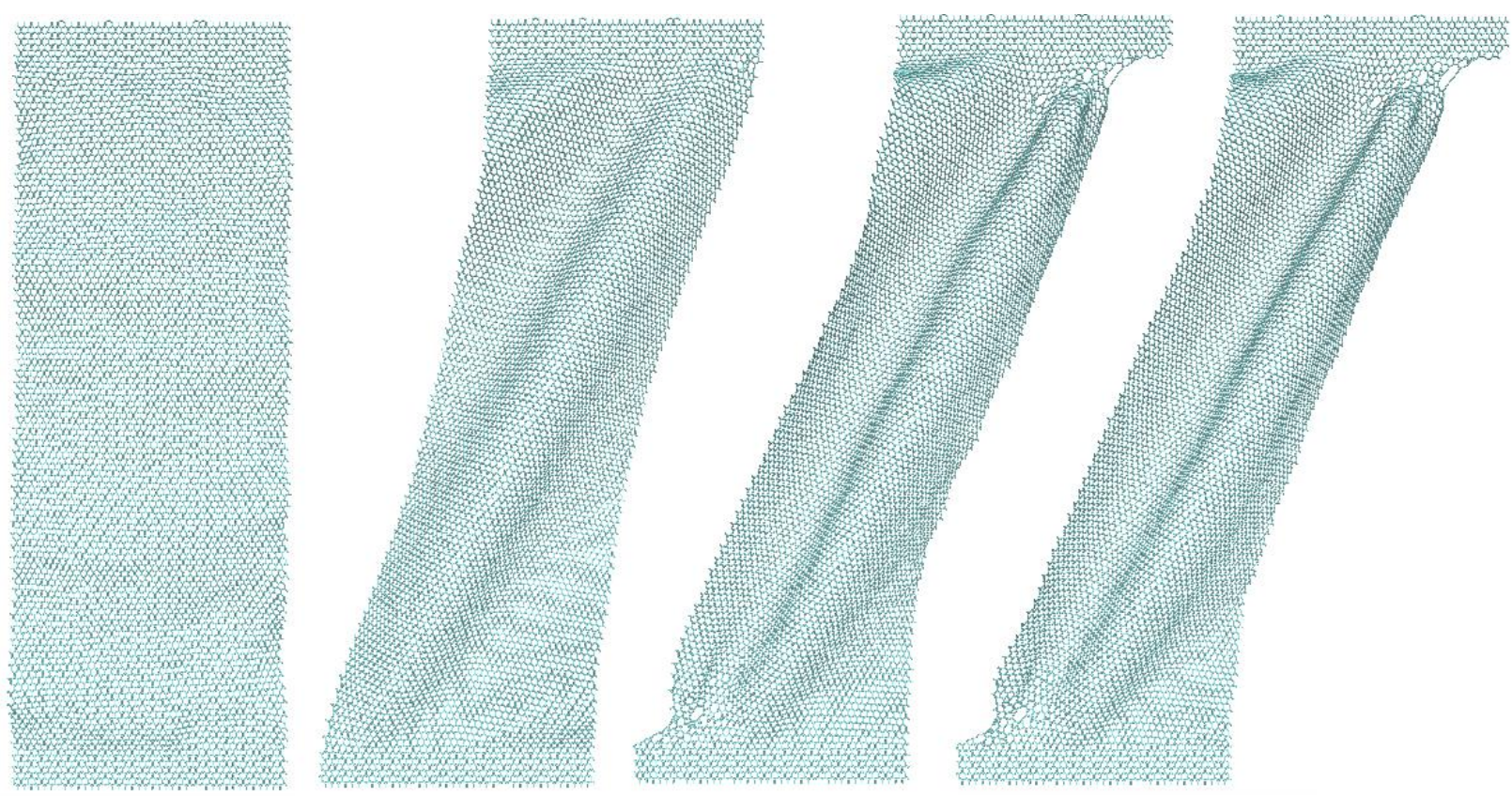

Figure 9 Evolution of the shear deformation in 25nm long armchair GNR until $40 \%$ strain

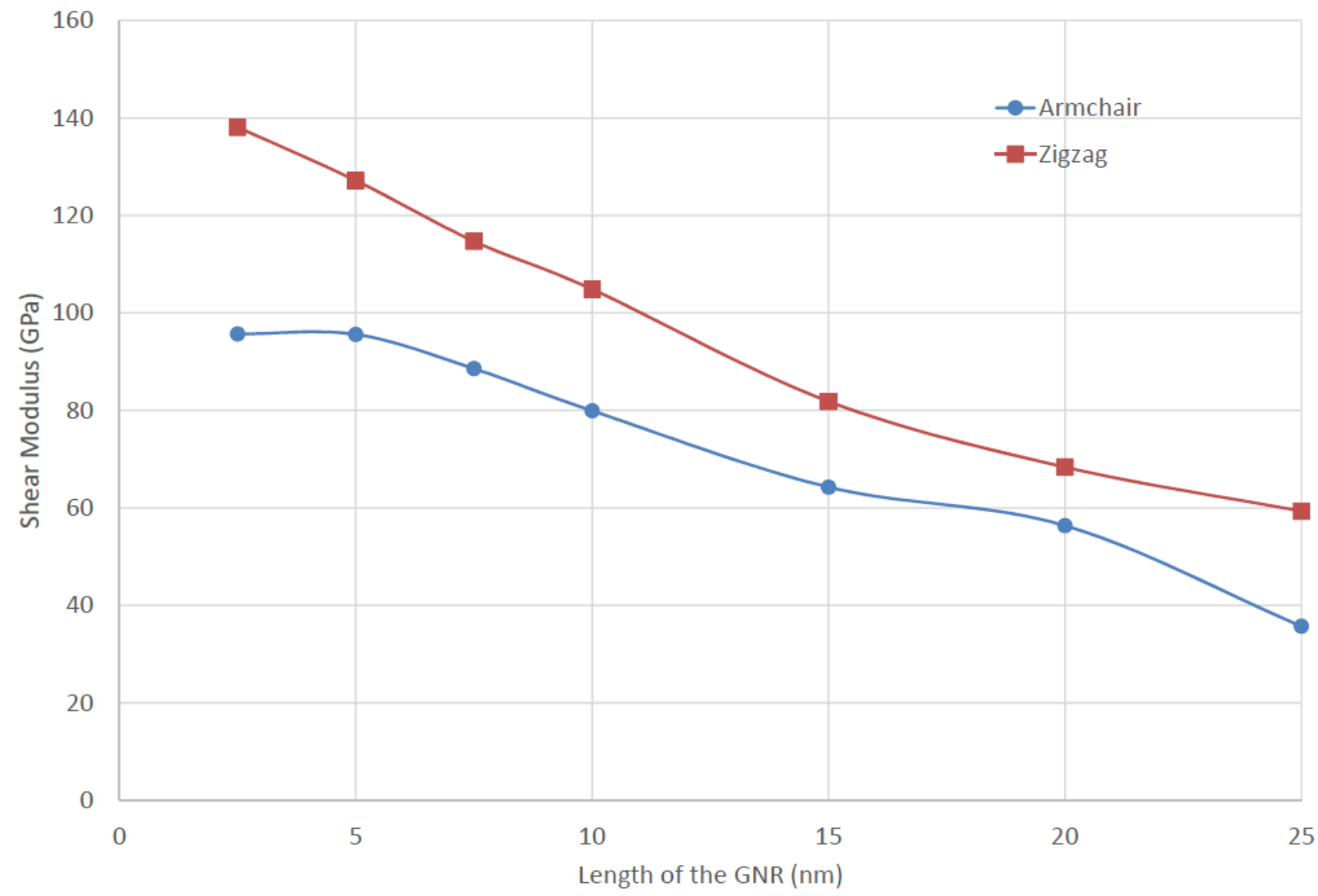

Figure 10 Shear Modulus of zigzag and armchair GNR as function of the length of the GNR 
In order to show a comparison of the behavior of zigzag and armchair GNR, the shear stressstrain curves of a sample GNR of $20 \mathrm{~nm}$ is shown in Figure 11. The shear modulus of zigzag GNR is always higher than that of armchair GNR. However, once the ultimate stress is reached and the softening starts, it is observed that armchair GNR are always stiffer in the softening zone than zigzag GNR. [The slope of the softening curve during that period is averaged, stiffness is defined as slope of the stress-strain curve post-ultimate stress]. This is due to the fact that the bond breakage stress-releasing phenomenon happen in zigzag GNR more often than in armchair GNR.

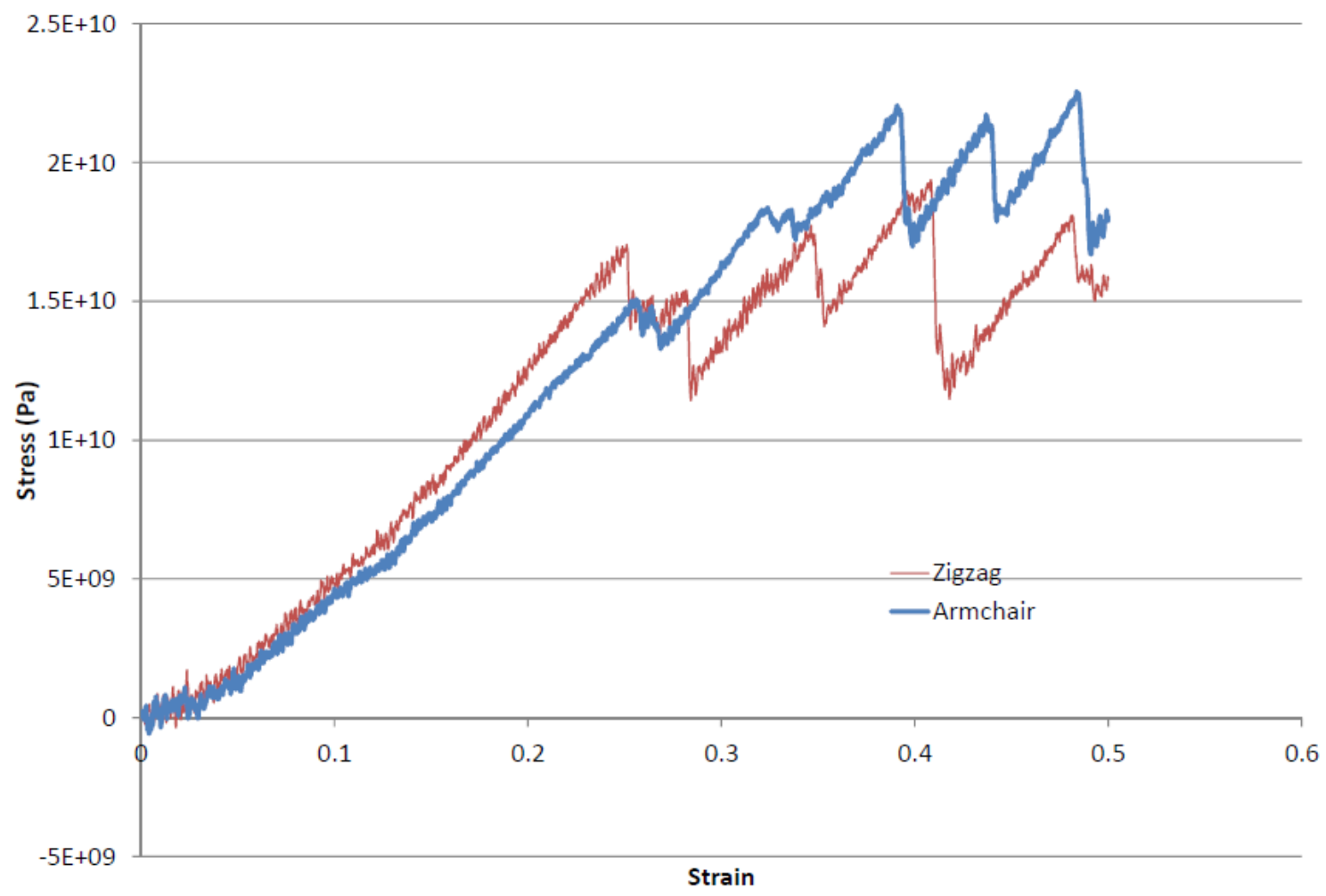

Figure 11 Shear stress-strain curves of $20 \mathrm{~nm}$ Zigzag and Armchair GNR

\section{Conclusions}

Molecular dynamics simulations were used to investigate the influence of the aspect ratio on the shear modulus and shear deformation behavior of armchair and zigzag graphene nanoribbons. 
Shear deformation applied was up to $400 \%$ strain for the lowest aspect ratio of 0.25 and up to $40 \%$ strain for the highest aspect ratio of 2.5. Results show that compared to square GNR, increasing the aspect ratio decreases the shear modulus significantly, especially for aspect ratio values of higher than one. Depending on the stress-relaxing phenomena, the average slope during softening increases with increasing GNR length. Moreover, in softening zone, stiffness is larger in armchair GNR than in zigzag GNR, but this effect is less profound at higher aspect ratios. It was observed that the ultimate elastic stress and its accompanying shear strain decreases as the GNR's size increases. Results show that GNR can sustain plastic shear deformations up to $400 \%$ strain without complete failure in wide GNR.

\section{Acknowledgments}

This project is sponsored by the United States Navy, Office of the Naval Research, Power Electronics program under the direction of Capt. Lynn Petersen. 


\section{References}

[1] K. S. Novoselov, A. K. Geim, S. V. Morozov, D. Jiang, Y. Zhang, S. V. Dubonos, et al., "Electric Field Effect in Atomically Thin Carbon Films," Science, vol. 306, pp. 666-669, October $22,20042004$.

[2] K. I. Bolotin, K. Sikes, Z. Jiang, M. Klima, G. Fudenberg, J. Hone, et al., "Ultrahigh electron mobility in suspended graphene," Solid State Communications, vol. 146, pp. 351-355, 2008.

[3] Y. Chu, T. Ragab, and C. Basaran, "Temperature dependence of joule heating in zigzag graphene nanoribbon," Carbon, vol. 89, pp. 169-175, 2015.

[4] B. Mortazavi, Z. Fan, L. F. C. Pereira, A. Harju, and T. Rabczuk, "Amorphized graphene: A stiff material with low thermal conductivity," Carbon, vol. 103, pp. 318-326, 7// 2016.

[5] A. A. Balandin, S. Ghosh, W. Bao, I. Calizo, D. Teweldebrhan, F. Miao, et al., "Superior thermal conductivity of single-layer graphene," Nano letters, vol. 8, pp. 902-907, 2008.

[6] Y. Chu, T. Ragab, P. Gautreau, and C. Basaran, "Mechanical properties of hydrogenedge-passivated chiral graphene nanoribbons," Journal of Nanomechanics and Micromechanics, vol. 5, pp. 04015001-04015008, 2015.

[7] Y. B. Chu, T. Ragab, and C. Basaran, "The size effect in mechanical properties of finitesized graphene nanoribbon," Computational Materials Science, vol. 81, pp. 269-274, 2014.

[8] T. Enoki, S. Fujii, and K. Takai, "Zigzag and armchair edges in graphene," Carbon, vol. 50, pp. 3141-3145, 8// 2012.

[9] H. Zhao, K. Min, and N. R. Aluru, "Size and Chirality Dependent Elastic Properties of Graphene Nanoribbons under Uniaxial Tension," Nano Letters, vol. 9, pp. 3012-3015, 2009/08/12 2009. 
[10] C. Lee, X. Wei, J. W. Kysar, and J. Hone, "Measurement of the Elastic Properties and Intrinsic Strength of Monolayer Graphene," Science, vol. 321, pp. 385-388, July 18, 20082008.

[11] R. Rasuli, A. I. zad, and M. M. Ahadian, "Mechanical properties of graphene cantilever from atomic force microscopy and density functional theory," Nanotechnology, vol. 21, p. $185503,2010$.

[12] F. Ricardo, A. D. Pablo, P. Helena, G. Cecilia, and W. M. Álvaro, "Mechanical properties of graphene nanoribbons," Journal of Physics: Condensed Matter, vol. 21, p. 285304, 2009.

[13] Q. Pei, Y. Zhang, and V. Shenoy, "A molecular dynamics study of the mechanical properties of hydrogen functionalized graphene," Carbon, vol. 48, pp. 898-904, 2010.

[14] K. Min and N. Aluru, "Mechanical properties of graphene under shear deformation," Applied Physics Letters, vol. 98, p. 013113, 2011.

[15] A. Udupa and A. Martini, "Model predictions of shear strain-induced ridge defects in graphene," Carbon, vol. 49, pp. 3571-3578, 2011.

[16] C. Wang, Y. Liu, L. Lan, and H. Tan, "Graphene wrinkling: formation, evolution and collapse," Nanoscale, vol. 5, pp. 4454-4461, 2013.

[17] A. H. Kheirkhah, E. S. Iranizad, M. Raeisi, and A. Rajabpour, "Mechanical properties of hydrogen functionalized graphene under shear deformation: A molecular dynamics study," Solid State Communications, vol. 177, pp. 98-102, 2014.

[18] Q. Zheng, Y. Geng, S. Wang, Z. Li, and J.-K. Kim, "Effects of functional groups on the mechanical and wrinkling properties of graphene sheets," Carbon, vol. 48, pp. 4315-4322, 2010. [19] M. Yaghoobi and G. Z. Voyiadjis, "Effect of boundary conditions on the MD simulation of nanoindentation," Computational Materials Science, vol. 95, pp. 626-636, 2014. 
[20] M. Yaghoobi and G. Z. Voyiadjis, "Atomistic simulation of size effects in singlecrystalline metals of confined volumes during nanoindentation," Computational Materials Science, vol. 111, pp. 64-73, 2016.

[21] G. Z. Voyiadjis and M. Yaghoobi, "Large scale atomistic simulation of size effects during nanoindentation: dislocation length and hardness," Materials Science and Engineering: A, vol. 634 , pp. 20-31, 2015.

[22] E. Dontsova and T. Dumitrică, "Nanomechanics of twisted mono-and few-layer graphene nanoribbons," The journal of physical chemistry letters, vol. 4, pp. 2010-2014, 2013.

[23] D. W. Brenner, O. A. Shenderova, J. A. Harrison, S. J. Stuart, B. Ni, and S. B. Sinnott, "A second-generation reactive empirical bond order (REBO) potential energy expression for hydrocarbons," Journal of Physics: Condensed Matter, vol. 14, pp. 783-802, 2002.

[24] H. J. C. Berendsen, J. P. M. Postma, W. F. v. Gunsteren, A. DiNola, and J. R. Haak, "Molecular dynamics with coupling to an external bath," The Journal of Chemical Physics, vol.

$$
\text { 81, pp. 3684-3690, } 1984 .
$$

[25] K. Mylvaganam and L. C. Zhang, "Important issues in a molecular dynamics simulation for characterising the mechanical properties of carbon nanotubes," Carbon, vol. 42, pp. 20252032, 2004.

[26] T. Ragab and C. Basaran, "A framework for stress computation in single-walled carbon nanotubes under uniaxial tension," Computational Materials Science, vol. 46, pp. 1135-1143, 2009.

[27] Y. Huang, J. Wu, and K. C. Hwang, "Thickness of graphene and single-wall carbon nanotubes," Physical Review B, vol. 74, p. 245413, 12/12/ 2006. 
[28] T. Ragab and C. Basaran, "The Unravelling of Open-Ended Single Walled Carbon Nanotubes Using Molecular Dynamics Simulations," Journal of Electronic Packaging, vol. 133, pp. 020903-020909, 2011. 

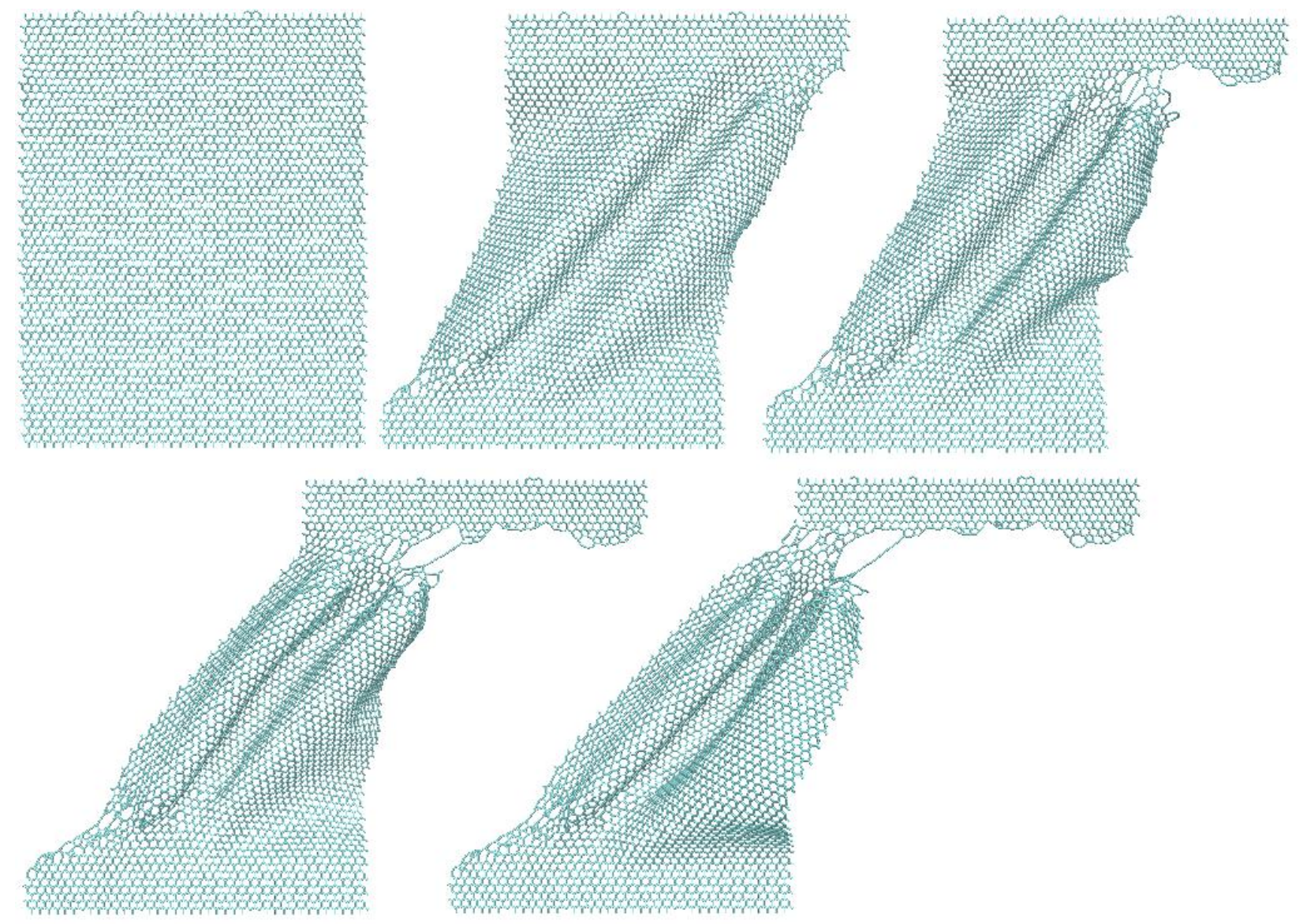